\title{
Neovascular glaucoma and diabetic macular edema in a patient with monolateral pseudoexfoliative glaucoma and type 2 diabetes: therapeutic effects of two-sided intravitreal triamcinolone acetonide treatment
}

\begin{abstract}
Background: To report on the safety and efficacy of intravitreal injections of triamcinolone acetonide in a patient with diabetic macular edema in the right eye and pseudoexfoliative glaucoma complicated by iris neovessels in the left eye.

Case presentation: Two intravitreal injections of triamcinolone acetonide were given (one per eye) in a 75 year-old caucasian male with macular edema in the right eye and neovascular glaucoma in the left eye. Changes in visual acuity, intraocular pressure, ophthalmoscopic examination of the anterior and posterior segment and flicker ERG response were regularly detected during a period of 6 months.

Conclusion: Bilateral visual acuity improvement was obtained after each injection and maintained for the whole observation period. Central retinal thickness in the right eye decreased after intravitreal injections of triamcinolone acetonide and remained stable until the last observation 6 months later. Iris neovessels in the left eye disappeared after the injection in the left eye. Flicker ERG implicit time in the right eye improved after intravitreal injections of triamcinolone acetonide, and remained stable in the left eye. No side effects of the treatment were observed. Intraocular pressure in the right eye remained within normal values without any intraocular pression-lowering drug, whereas it showed even some decrease in the left eye, despite the presence of glaucoma.
\end{abstract}

Volume 3 Issue 3 - 2015

\author{
Maria Giulia Mutolo, Claudia Fabiani, Nicola \\ Pescosolido \\ Rome University La Sapienza, Italy
}

\begin{abstract}
Correspondence: Maria Giulia Mutolo, Faculty of Medicine and Dentistry, Az Ospedaliera Sant Andrea, Rome University La Sapienza,Via di Grottarossa, I037 00189 Roma, Italy,
\end{abstract} Tel 00393394083914 ,Email mariagiuliamutolo@gmail.com

Received: October 23, 2015 | Published: November 13, 2015

\section{Abbreviations: DM, diabetes mellitus; DME, diabetic macular edema; NVG, neovascular glaucoma; NVs, angle neovessels; IVTA, intravitreal injections of triamcinolone acetonide; RE, right eye; LE, left eye; NCVA, non-corrected visual acuity; CF, count fingers; OCT, optical coherence tomography}

\section{Background}

Progressive ocular complications of diabetes mellitus (DM) result by the chronic exposure to high blood levels of glucose. Diabetic macular edema (DME) is the most common complication, and the cause of moderate to severe visual loss in diabetic patients. Furthermore, DM is the major etiologic factor for neovascular glaucoma (NVG) [1]. The association of iris and angle neovessels (NVs) with DM becomes more frequent with the duration of the disease and inadequate blood sugar control. ${ }^{2}$ In the case here reported, the patient was affected by pseudoexfoliative glaucoma in his left eye, and by DME in the right eye. Intravitreal steroids are have been used in the management of both DME and NVG, due to their antiinflammatory and anti angiogenic effects. Indeed, several studies reported the benefits of triamcinolone acetonide intravitreal injections (IVTA) in reducing DME and increasing visual acuity, ${ }^{3-6}$ alone or in association with laser treatment, and also like a useful additional tool in the management of neovascular glaucoma. ${ }^{7-9}$

\section{Case presentation}

A 75-year-old Caucasian male patient, with type II diabetes and hypertension (under oral therapy with hypoglycemic drugs) presented with DME in the right eye (RE) and iris and angle NVs in the left eye (LE). The patient had pseudoexfoliative glaucoma in the left eye and was on anti glaucoma treatment with an association of timolol and brimonidine plus brinzolamide. Non corrected visual acuity (NCVA) was 20/400 in the RE and count fingers (CF) in the LE. Intraocular pressure (IOP) was $18 \mathrm{mmHg}$ in the RE and $36 \mathrm{mmHg}$ in the LE. Figure 1 shows iris neovascularization of the left eye at slit lamp examination before and after IVTA treatment.

Figure 2 shows the optical coherence tomography (OCT) radial scan of the macula before and after IVTA treatment. Figure 3 shows the trend of macular thickness (a), flicker ERG implicit time (b) and IOP (c). Slit lamp examination of the RE anterior segment revealed moderate lens opacity while in the LE it showed iris and angle NVs, fixed mydriasis, pseudoexfoliation of lens capsule and mild lens opacity. The fundus examination of both eyes showed diabetic 
retinopathy with hemorrhages, microaneurysms, hard exudates and clinically significant macular edema in the RE with subfoveal and perifoveal hard exudates. Macular thickness measured with the OCT

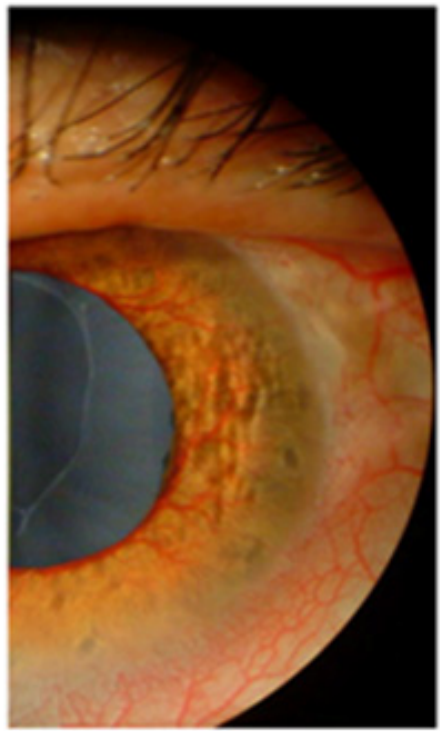

a

was $634 \mu \mathrm{m}$ in the RE and $200 \mu \mathrm{m}$ in the LE. Flicker ERG implicit time was $36.64 \mathrm{~ms}$ in the RE and $42.15 \mathrm{~ms}$ in the LE.

Figure I Morphological analysis of the left eye.A) Picture of the anterior segment soon before IVTA; B) Picture of the anterior segment two months after IVTA.

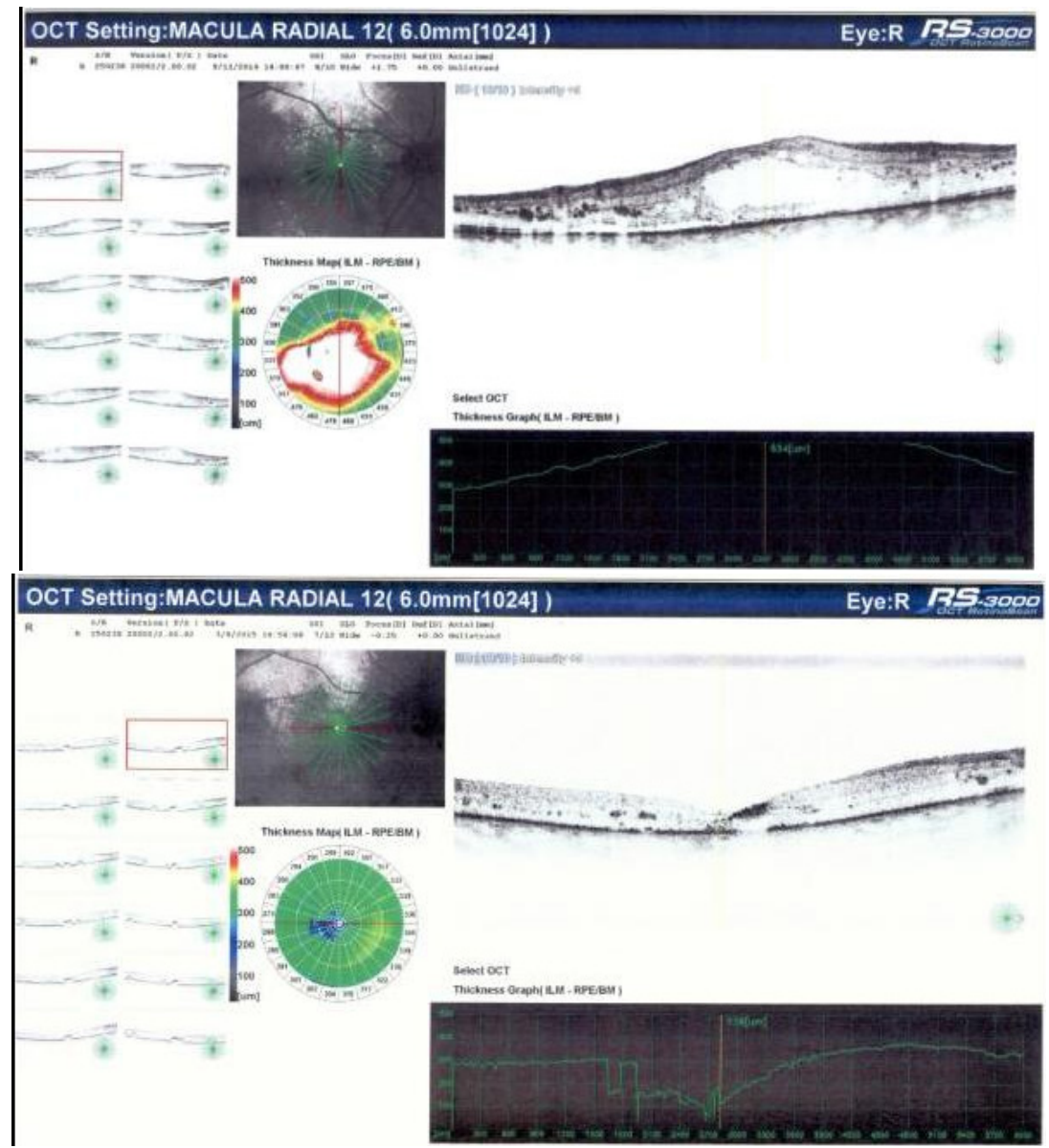

Figure 2 Morphological analysis of the right eye.A) OCT macular thickness soon before IVTA; B) OCT macular thickness 6 months after IVTA. 
$\mu \mathrm{m} 700$

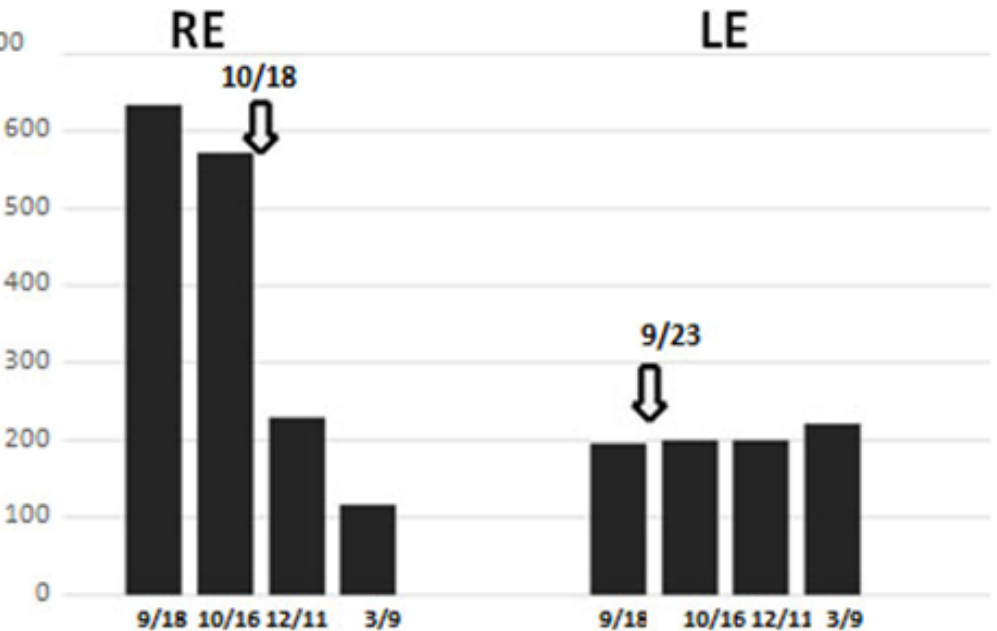

$\mathrm{ms}$

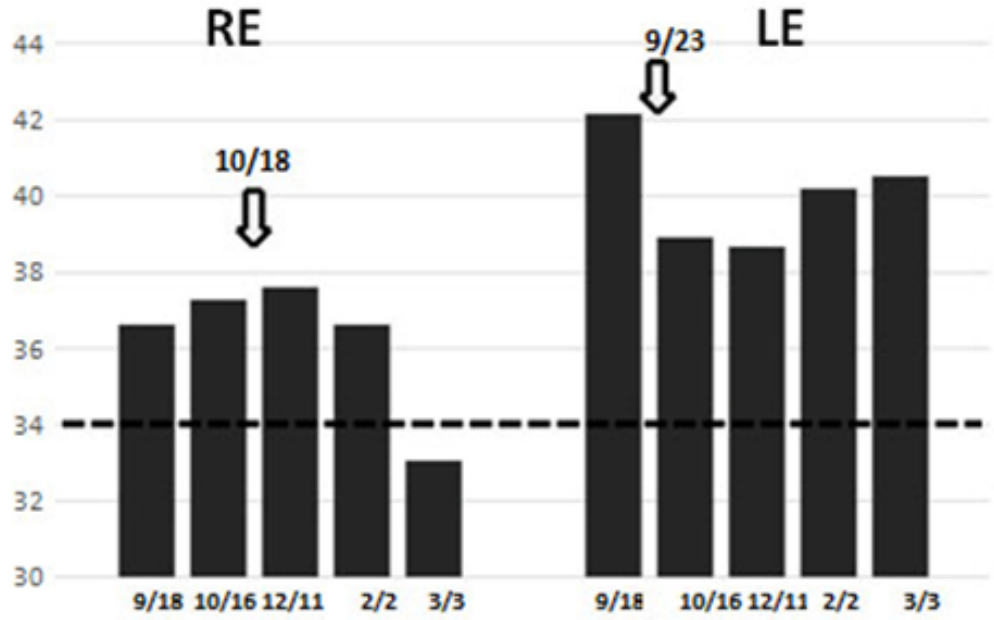

$\mathrm{mmHg} 45$

RE

LE

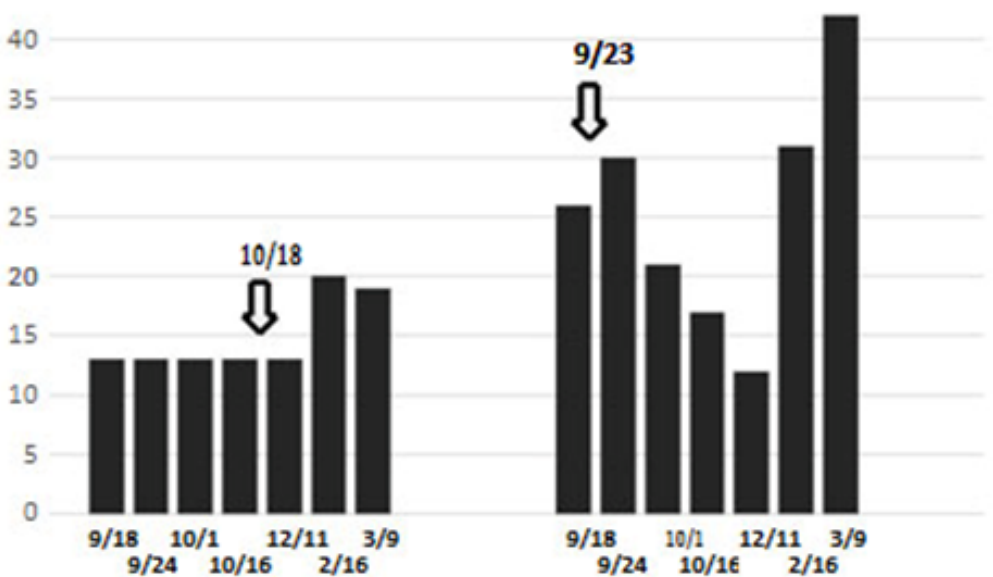

a

b 
An IVTA was proposed for both eyes commencing with the LE. Therefore, a first injection of $4 \mathrm{mg}(0.05 \mathrm{ml})$ triamcinolone acetonide (Taioftal ${ }^{\circledR}$, Sooft Italia S.p.A, IT) was performed in the LE. After one month, visual acuity was still $\mathrm{CF}$, but iris and angle NVs were barely visible both at slit lamp examination and gonioscopy. IOP was reduced to $17 \mathrm{mmHg}$ and the fundus examination showed a marked reduction of the hard exudates. After two months followup, BCVA in the LE increased to 20/100 and no iris and angle NVs were detectable at slit lamp examination. IOP further decreased to $12 \mathrm{mmHg}$. Hard exudates were strongly decreased at fundoscopy, OCT macular thickness was $196 \mu \mathrm{m}$ and flicker ERG implicit time decreased to $38.50 \mathrm{~ms}$. After six months follow-up, BCVA remained stable at 20/100 while IOP returned to $42 \mathrm{mmHg}$. Iris and angle NVs reappeared and were detectable both at slit lamp examination and gonioscopy. Fundus examination showed no reappearance of hard exudates, OCT macular thickness was stable at $221 \mu \mathrm{m}$, whilst flicker ERG implicit time returned to $40.53 \mathrm{~ms} .25$ days after the IVTA in the LE, an injection of $4 \mathrm{mg}(0,05 \mathrm{ml})$ triamcinolone acetonide (Taioftal (R) was given also in the RE. At one month follow- up NCVA was still 20/400, IOP was $13 \mathrm{mmHg}$ and OCT macular thickness already decreased to $229 \mu \mathrm{m}$. At fundus examination subfoveal and perifoveal hard exudates were reduced together with the entity of DME. Flicker ERG implicit time was $37.61 \mathrm{~ms}$. Five months after treatment, visual acuity was improved to $20 / 100$, IOP was $20 \mathrm{mmHg}$ and fundus examination revealed a further reduction of the hard exudates and of the DME (macular thickness $116 \mu \mathrm{m}$ ). Flicker ERG implicit time decreased to $33.07 \mathrm{~ms}$.

\section{Discussion}

This case illustrates the usefulness, safety and efficacy of intravitreal injections of triamcinolone acetonide in a patient with diabetic macular edema in the right eye and pseudoexfoliative glaucoma complicated by iris and angle neovessels in the left eye. During the 6 months observation period all the parameters under analysis were improving. Most notably, OCT showed a rapid important thinning of the macular area in the RE. Flicker ERG implicit time, as demonstrated in previous studies, ${ }^{10,11}$ is a quantifiable objective measurement of photoreceptor damage in different types of retinal diseases. In this case, its initial values were above the normality threshold of $34 \mathrm{~ms}^{10}$ in both eyes. After IVTA in the RE we observed a decrease of its value below the normality threshold $(33.07 \mathrm{~ms}$ at 6 months) that was concomitant with the normalization of macular thickness at $116 \mu \mathrm{m}$ and a modest increase of visual acuity. In contrast, flicker ERG values in the glaucomatous LE remained stable, above the normality threshold, despite some improvement of the visual acuity.

\section{Conclusion}

In good agreement with the known literature, ${ }^{3-6,12}$ IVTA showed a good efficacy in both decreasing macular thickness in the RE and iris and angle NVsin the LE. No complications were observed during the whole 6 months observation period and the procedure resulted in rapid improvement of most of the symptoms. Furthermore, the steroidal treatment has shown to be effective in both the pathological conditions, and this is probably due to the inflammatory etiopathogenesis of both diseases. NVG, in fact, is a secondary ocular pathological condition resulting from a myriad of ocular and systemic conditions with retinal ischemia as a mediator in over $95 \%$ of cases. Inflammatory process maybe an important cause of iris neovascularization in subjects with NVG in addition to growth factors alone. In addition, DR manifests characteristics of both vasculopathy and chronic inflammatory diseases. Therefore, inhibition of the inflammatory process represents a therapeutic strategy valid for both diseases. The risks related to the intravitreal injection of steroids seem to be reasonable and well manageable. The IOP increase is higher in eyes with glaucoma and a high baseline IOP, ${ }^{13}$ although it appears to be manageable by anti glaucoma drugs. ${ }^{14}$ However, in this patient no further increase of IOP was observed, rather IOP in the both eyes even showed some decrease in the weeks following IVTA. Cataract progression is also known to occur as a consequence of steroid injection, ${ }^{15}$ but it requires longer times of observation, falling outside the observation time reported for this patient.

\section{Acknowledgments}

None.

\section{Conflicts of interest}

The author declares there is no conflict of interest.

\section{References}

1. Al-Shamsi HN, Dueker DK, Nowilaty SR, et al. Neovascular glaucoma at king khaled eye specialist hospital - etiologic considerations. Middle East Afr J Ophthalmol. 2009;16(1):15-19.

2. Morrison JC, Pollack IP. Neovascular glaucoma (Chapter 21). Glaucoma Science and Practice, $1^{\text {st }}$ ed. Thieme Medical Publishers, New York, USA, 2003. p. 226-236.

3. Audren F, Erginay A, Haouchine B, et al. Intravitreal triamcinolone acetonide for diffuse diabetic macular oedema: 6-month results of a prospective controlled trial. Acta Ophthalmol Scand. 2006;84(5):624 630 .

4. Lambiase A, Abdolrahimzadeh S, Recupero SM. An update on intravitreal implants in use for eye disorders. Drugs Today (Barc). 2014;50(3):239249.

5. Diabetic Retinopathy Clinical Research Network, Elman MJ, Aiello LP, et al. Randomized trial evaluating ranibizumab plus prompt or deferred laser or triamcinolone plus prompt laser for diabetic macular edema. Ophthalmology. 2010;117(6):1064-1077.e35.

6. Gillies MC, Sutter FK, Simpson JM, et al. Intravitreal triamcinolone for refractory diabetic macular edema: two-year results of a doublemasked, placebo-controlled, randomized clinical trial. Ophthalmology. 2006;113(9):1533-1538.

7. Collignon NJ, Crommen J, Collignon-Brach J, et al. Low dose intravitrealtriamcinolone acetonide in the treatment of neovasularglaucoma. Bull SocBelgeOphtalmol. 2005;(298):5-10.

8. Jonas JB, Kreissig I, Degenring RF. Neovascular glaucoma treated by intravitreal triamcinolone acetonide. Acta Ophthalmol Scand. 2003;81(5):540-541.

9. Scuderi GL, Pasquale N. Laser therapies for glaucoma: new frontiers Prog Brain Res. 2008;173:225-236.

10. Pescosolido N, Fazio S, Rusciano D. Reliability of the implicit time of flicker ERG B-Wave a san objective diagnostic tool for retinopathies. JSM Biotechnology \& Biomedical Engineering. 2014.

11. Pescosolido N, Fazio S, Rusciano D. Therapeutic improvement in the contralateral eye after ranibizumab intravitreal treatment in a patient affected by bilateral subfoveal choroidal neovascularization. JSM Biotechnology \& Biomedical Engineering. 2014.

12. Bonanomi MT, Susanna R. Intravitreal triamcinolone acetonide as adjunctive treatment for neovascular glaucoma. Clinics (Sao Paulo). 2005;60(4):347-350. 
13. Vasconcelos-Santos DV, Nehemy PG, Schachat AP, et al. Secondary ocular hypertension after intravitreal injection of $4 \mathrm{mg}$ of triamcinolone acetonide: incidence and risk factors. Retina. 2008;28(4):573-580.

14. Grover D, Li TJ, Chong CC. Intravitreal steroids for macular edema in diabetes. Cochrane Database Syst Rev. 2008;(1):CD005656.
15. Martidis A, Duker JS, Greenberg PB, et al. Intravitreal triamcinolone for refractory diabetic macular edema. Ophthalmology. 2002;109(5):920927.

Citation: Mutolo MG, Fabiani C, Pescosolido N. Neovascular glaucoma and diabetic macular edema in a patient with monolateral pseudoexfoliative glaucoma and type 2 diabetes: therapeutic effects of two-sided intravitreal triamcinolone acetonide treatment. Adv Ophthalmol Vis Syst. 20I5;3(3):288-292. 